Received on: 15.12.2016

Revised on: 31.12.2016

www.ijoaem.com

\title{
Advance Agro Farm Design With Smart Farming, Irrigation and Rain Water Harvesting Using Internet of Things
}

\author{
Authors: \\ Vinod Sukhadeve \\ NIT Yupia, Arunachal Pradesh, India \\ e-mail:vin3yasukhadeve@gmail.com \\ Sahadev Roy \\ Dept. of ECE, NIT Arunachal Pradesh, Yupia, 791112, India \\ e-mail:sdr.ece@nitap.in
}

Subject Category: Engineering

Sub Category: Internet of Things

Editor:

Arindam Biswas

Volume 1 Issue 1 December 2016

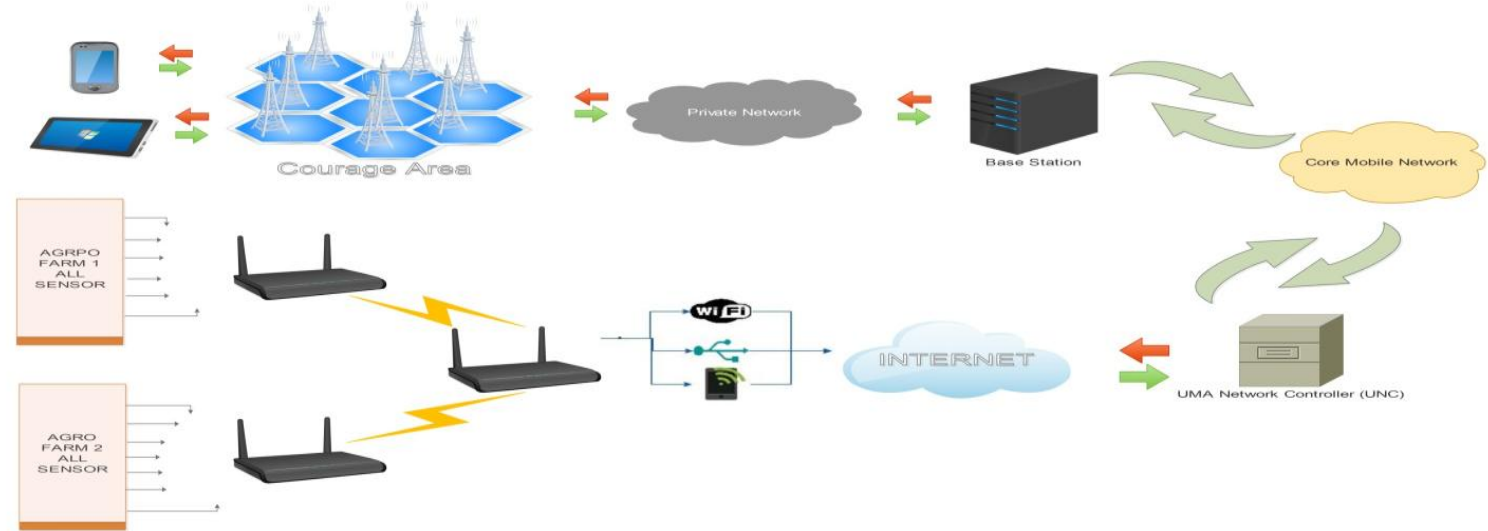

Connection of sensor devices with help of Unlicensed Mobile Access (UMA) and GAN-Generic Access Network.

Keywords: $\quad$ Internet of things(IoT);

Agriculture;

Irrigation;

Wi-Fi;

Pest control;

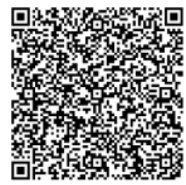

IJOAEM/5/1/1/5

Vinod Sukhadeve and Sahadev Roy "Advance Agro Farm Design With Smart Farming, Irrigation And Rain Water Harvesting Using Internet of Things," International Journal of Advanced Engineering and Management, Vol. 1, No. 1, pp.33-45, 2016. 


\title{
Advance Agro Farm Design With Smart Farming, Irrigation and Rain Water Harvesting Using Internet of Things
}

\author{
Vinod Sukhadeve and Sahadev Roy
}

\begin{abstract}
The paper presents the design of agriculture farm especially for the plane region which can well utilize by the farmer to sort out the scarcity of water for crop growth. The farmers are subjected with the lots of problem in agriculture like improper irrigation, selection of crops, non availability of whether information according to their region, the problem from pest and wild animals. Due to these problems, the suicidal case of farmers gets increase day by day. These problems can be sort out by using IoT. Here we use Arduino Yun having inbuilt Wi-Fi to transfer and analyze data using any IoT platform likes Kaa IoT, Watson IoT, and Cayenne. We can use different IoT communication technology like Z-wave, 6LowPAN, Thread, Sigfox, and Neul to communicate various sensors to the external world according to the application. Here we simulate the design of entire sensor network used in this project using NetSim simulator and emulator software. After emulation of designed network design by taking $50 \mathrm{~m}$ as field size, we obtained various graphs which show throughput of each link from sensor node up to the monitoring base station, graphs of various parameter like packet transfer, collided packets, payload and overhead transmitted and battery consumed by each sensor for specified duration. Also, farmers are able to grow a health hazard free crop for the upcoming generation.
\end{abstract}

\section{Introduction}

To observe and handle anything from anywhere electronically, the internet plays a very vital role .Today's world is based on the internet. People wants to observe their work from anywhere on their digital devices such as Smartphone and tablet or laptop. Several things were made easy by using Internet of Thing (IoT)[1]. Internet of things is a network of surrounding physical devices, vehicles, devices related to electronics, embedded software, and hardware. Connectivity networks will help them to collect and exchange of data over a globe. We can compare the obtained data with the specified area location and previous data analysis or any pre-defined standard. IoT helps or allows the sensed data to monitor as well as control from anywhere in the world, where the internet connectivity available. So this types of a system result in the improvement in efficiency, accuracy and help the economy to achieve a great peak in this digital world. The IoT is somewhat a hot topic in many industries as in latest trending technology, but it is not a new concept actually. In the early 2000's, Kevin Ashton proposed the first theory on IoT. $\mathrm{He}$ is also known as the father of Internet of Things. He did very good job in the field of RFID and how to transfer the data collected from RFID to the internet. To handle anything from anywhere electronically, the internet plays a very vital role .Today's world is based on the internet. Fig.1 signifies the recent trend in IoT.

\section{a) The Main Problems Are Associate with the Farmers}

Crops need sufficient water which is not available whenever required. For pest control, lots of money gets wasted in buying pesticides, adverse effect on human health. Farmers not able to use water efficiently. Farmers need to go to the respective agriculture farm for observing the water level, humidity level as well as soil moisture. Sometimes, the farmer is not present at the farm; at that time wild animal generally attacks crops, so huge losses occur.

\section{b) Solutions to the Problem}

Multiple crops, need to modernize agriculture, proper irrigation technique by rainwater harvesting. By using the concept of the internet of things, we can avoid these types of problem in the future. This can be done by modifying our current farm system which applied to the plane region only.

Vinod Sukhadeve and Sahadev Roy "Advance Agro Farm Design With Smart Farming, Irrigation And Rain Water Harvesting Using Internet of Things," International Journal of Advanced Engineering and Management, Vol. 1, No. 1, pp.33-45, 2016. 


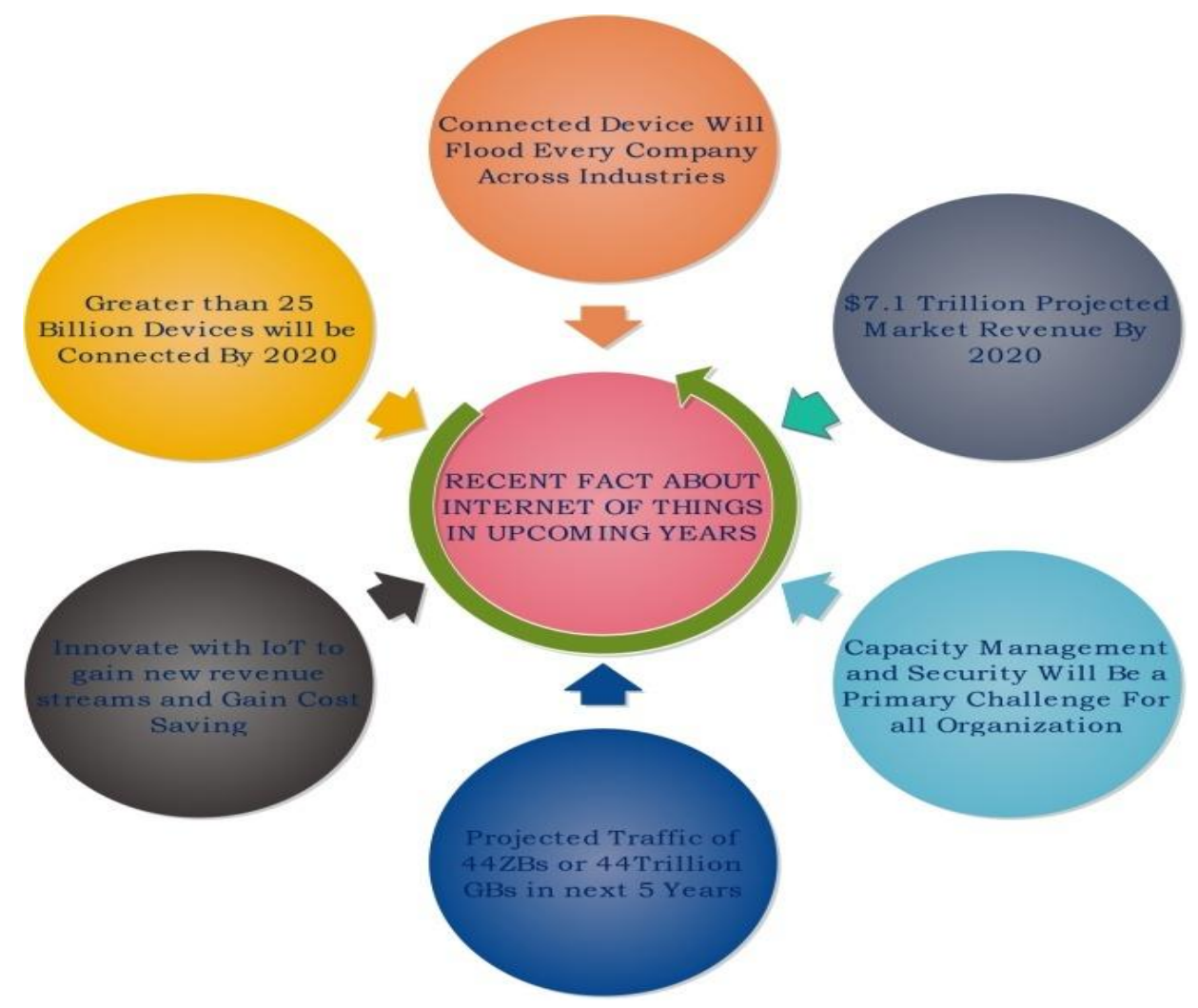

Figure 1. Technology chart, which signifies the recent trends in IoT.

\section{Proposed Work}

It can be done by modifying current farm system used in the plane region, which consists of farms arranged in the staircase manner and at the bottom one water tank is present. To carry out excess water from one farm to another, a small opening is provided. The entire excess water is finally collected by the water tank which is well fabricated to store enough water to satisfy water requirement by using various types of irrigation technique. The tank also fills up by rain water.

\section{a) The Designed Agro Farm Structure is as Follows}

The working procedure of above-proposed model is explained in Table1. The selection of various crops by farmer depends on the types of seasons. Based on that farmer will choose the best crop which satisfies all the growing condition of the crop taken under consideration. The excess water which not required is collected from one farm to 2nd and second farm to third through the small opening, which is controlled by actuator valve. The amount of water discharge from one form to other depends on the crop of the respective farm (humidity level in soil determine the types of crop used in the respective farm). All the water is collected in the water tank as shown in the structure. The tank also received rainy ground water. The water is then utilized for maintaining the moisture level in the soil according to the crop in the respective farm. During the rainy season, we get only 100lakh Lit/hectare of water through $100 \mathrm{~cm}$ rainfall, but Agriculture farm needed only 30 lacks Lit/hectare for the satisfactory growing of any plant. But what happened if any area is subjected to less rainfall. As per study rainfall is not the only risk factor. If we calculate the water distribution with respect to the agriculture field, nearly 5\% water evaporates due to tremendous heat produced by the earth atmosphere (Fig. 2). Several factors are responsible for the heat generation such as global warming, deforestation caused because of the greenhouse effect. Nearly $5 \%$ again lost as runoff from the surface, due to several soil properties such as soil texture, infiltration, Hydraulic, water-holding capacity, and Water logging associated with low soil permeability [2]. The rest part of water i.e.40-50\% is absorbed by the soil which provides nutrition content to the plant required to sustained plant growth. When soil absorbed water as moisture, remaining water i.e. $30-40 \%$ will be added to ground when the field capacity reached its water holding capacity.

Vinod Sukhadeve and Sahadev Roy "Advance Agro Farm Design With Smart Farming, Irrigation And Rain Water Harvesting Using Internet of Things," International Journal of Advanced Engineering and Management, Vol. 1, No. 1, pp.33-45, 2016. 
Irrigation plays a very important role in expanding the production of various food crops. The food production is directly dependent on the quality of irrigation provide to the crops. We can get $55 \%$ of agricultural output from irrigated lands only so, it is necessary to give more stress on the irrigation method for solving water scarcity problems.

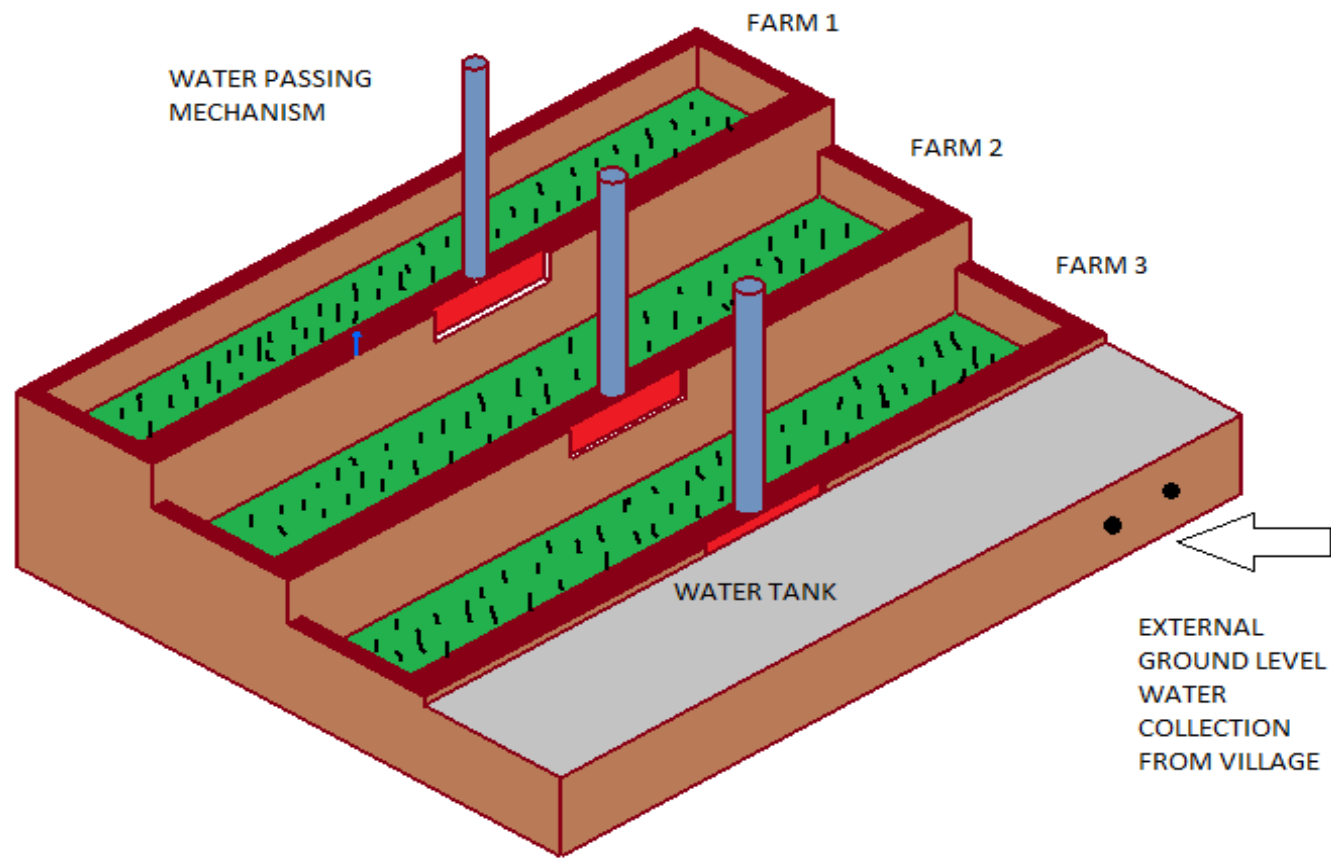

Figure 2: Proposed design of agriculture farm for plane region

Table 1. Working Procedure of Proposed Model

\begin{tabular}{|c|c|c|c|}
\hline Farm 1 & Farm 2 & Farm 3 & Water Tank \\
\hline $\begin{array}{l}\text { The crop which required less } \\
\text { water grows here. }\end{array}$ & $\begin{array}{l}\text { The crop which required less } \\
\text { water than Farm } 1 \text { and more } \\
\text { than Farm } 3 \text { grows here. }\end{array}$ & $\begin{array}{l}\text { The crop which required more } \\
\text { than farm } 1 \text { and farm } 2 \text { should } \\
\text { grow here. }\end{array}$ & $\begin{array}{l}\text { The extra water from Farm } 1,2,3 \\
\text { will diverted to the water tank. } \\
\text { This water is utilized to feed back } \\
\text { to respective farm whenever } \\
\text { required. }\end{array}$ \\
\hline
\end{tabular}

a) Several Factors Affect the Cause of Less Production Due To Water Deficiency which is listed below.

I. Decline in rainfall - The most commonly discussed cause of drought

II. Water evaporation- As global temperatures continue to rise, water will evaporate faster [3].

Most of the water utilized for agriculture purpose, industries, and household use. Our natural heritage like rivers, seas and oceans have been exploited, maltreated and contaminated. Water distribution in the farming fields is shown in Table 2.

Table 2. Typical water utilization table

\begin{tabular}{|c|c|c|}
\hline Sr No & \% of water out of $\mathbf{1 0 0} \%$ & Distribution of water \\
\hline 1 & $0-5 \%$ & Gas evaporated annually \\
\hline 2 & $0-5 \%$ & Loss as a run off \\
\hline 3 & $40-50 \%$ & Becomes soil moisture for crop \\
\hline 4 & $30-40 \%$ & Adds to ground water after reaching field capacity \\
\hline
\end{tabular}

Vinod Sukhadeve and Sahadev Roy "Advance Agro Farm Design With Smart Farming, Irrigation And Rain Water Harvesting Using Internet of Things," International Journal of Advanced Engineering and Management, Vol. 1, No. 1, pp.33-45, 2016. 


\section{b) Different Types Of Irrigation Methods Deployed At Agricultural Farm Are As Follows:}

I. Sprinkler irrigation system,

II. Drip irrigation system,

III. Tank modernization,

IV. Farmers participation,

V. Reuse of wastewater,

VI. Inter basin transfer.

Out of the above-mentioned method, drip irrigation system saves more water i.e. $40-70 \%$, and also increase the production rate from $10-100 \%$.but in the case of sprinkler irrigation system, only $30-40 \%$ of water is saved. Therefore drip irrigation method is used by many farmers. We employ drip irrigation system in this designed agro farm structure for the plane area.

\section{c) Irrigation Efficiency Calculation}

We can find out irrigation efficiency which is usually expressed as the ratio of the amount of water used for crop growing plus water that maintain necessary salt balance in the crop root zone to the total volume of water provided to the farm.

We designed the sophisticated irrigation system to enhance the water utilization factor of the field, which is decided by the efficiency. The overall efficiency which is also known as irrigation efficiency is expressed as follows:

$$
\begin{aligned}
& E_{\text {irrigation }}=\text { Total }_{\text {water suplied }}-\text { water }_{\text {aborbed by farm }}-\text { total }_{\text {water run off }}-\text { Loss }_{\text {in pipeline }} \times 100 \\
& \text { Total }{ }_{\text {water suplied }} \\
& \text { Where, } \boldsymbol{E}_{\text {irrigation }}=\text { irrigation efficiency. } \\
& \text { Total }_{\text {water suplied }}=\text { Amount of water supplied to the agro farm. } \\
& \text { water }_{\text {aborbed by farm }}=\text { amount of water absorbed by crops. } \\
& \text { total }_{\text {water run off }}=\text { amount of water runoff from farm. } \\
& \text { Los }_{\text {in pipeline }}=\text { losses in pipelines as well as in channel } \\
& \text { Reservoir storage efficiency calculation: }
\end{aligned}
$$

In advance designing of agro farm for plane region, we need to setup water reservoir to meet the water scarcity problems. The reservoir storage efficiency is expressed as:

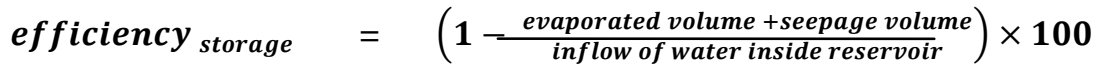

Where, Seepage volume is the slow escape of water through porous material or small holes.

The crops in the farm suffered from pest and insects. According to the report presented by the WHO, due excessive use of pesticide nearly 22000 deaths happening each year in developing countries. So without using any pesticide, we can control pest by using the advanced technique, which is non-hazardous to human health and also environmental friendly. In this technique, we will use ultrasound of low frequency to high (frequency depends on crop).This sound is not audible to the human being but it acts as pest repellent EM wave.

Also, another main problem is animals which are come to destroy the crop. The group of animals such as cattle, elephant, pig, and the monkey is afraid of the sound of lions, tiger etc. by using Arduino mp3 shield we can produce dangerous sound whenever monkeys come in contact with the crop. We can implement internet of things in smart agriculture purpose like shown in Fig. 3. To get all the live data of fields like soil moisture, soil humidity, temperature, wind speed, area wise rainfall, soil PH and the water level of the agriculture farm during the rainy season we need different sensors according to the application. Now it's time to connect all the sensors to the external world, to do this we require various protocol like ZigBee, WI-Fi, Bluetooth, cellular, thread etc. Based on the type of application and distance from sensor location to the router, we have to choose protocol accordingly. In the above-shown diagram, we used Arduino Yun which is having inbuilt Wi-Fi with various status indicator led as shown in Fig. 5 for connecting the entire sensor to the external world.

Vinod Sukhadeve and Sahadev Roy "Advance Agro Farm Design With Smart Farming, Irrigation And Rain Water Harvesting Using Internet of Things," International Journal of Advanced Engineering and Management, Vol. 1, No. 1, pp.33-45, 2016. 


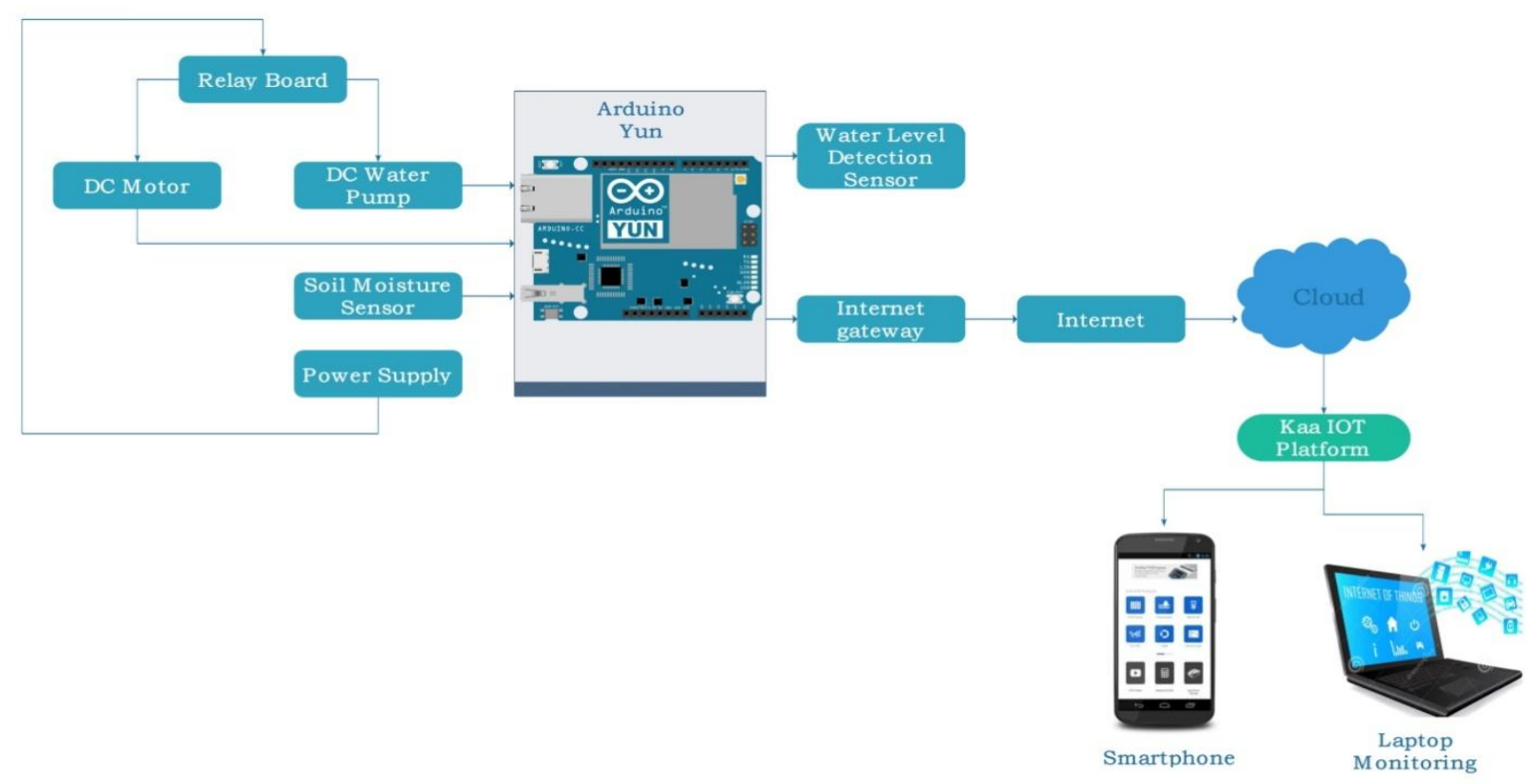

Figure 3. Block diagram of connection of various sensors through internet by using Arduino Yun.

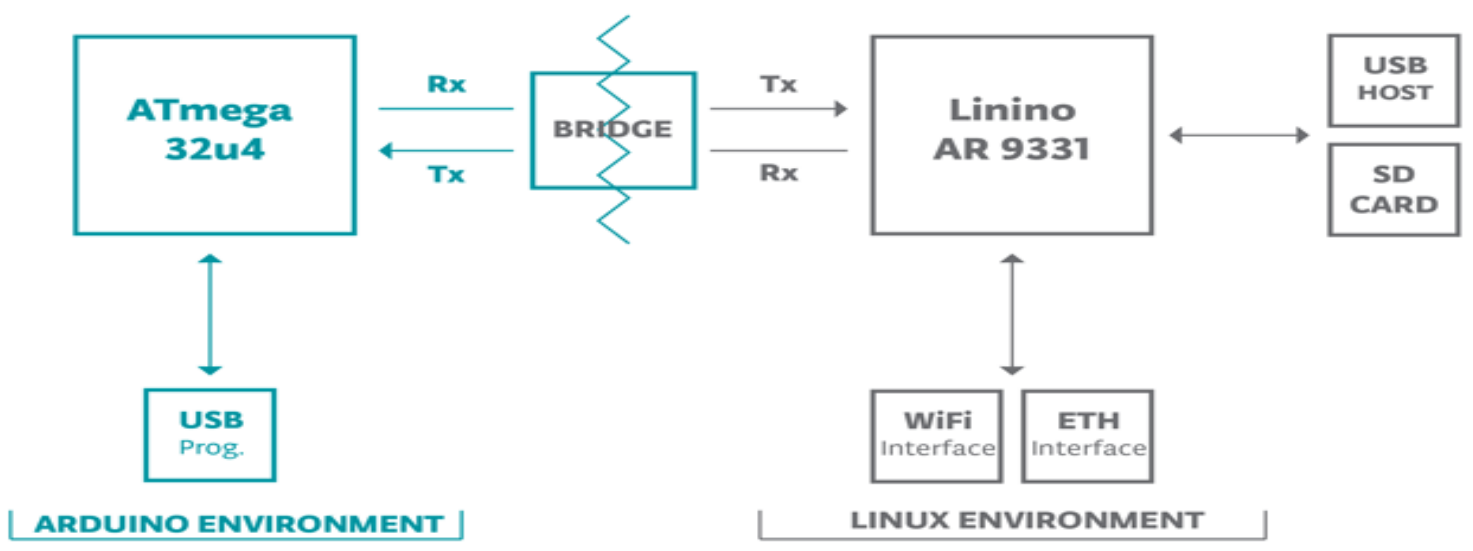

Figure 4. The bridge library enables communication between Arduino And Openwrt-Yun[4].

Vinod Sukhadeve and Sahadev Roy "Advance Agro Farm Design With Smart Farming, Irrigation And Rain Water Harvesting Using Internet of Things," International Journal of Advanced Engineering and Management, Vol. 1, No. 1, pp.33-45, 2016. 


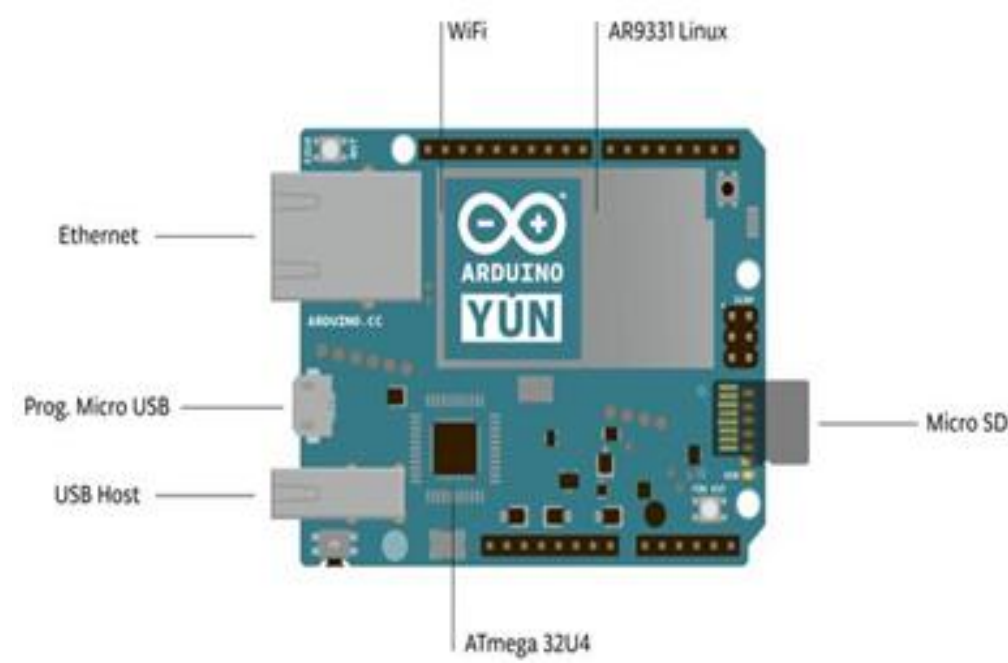

(a)

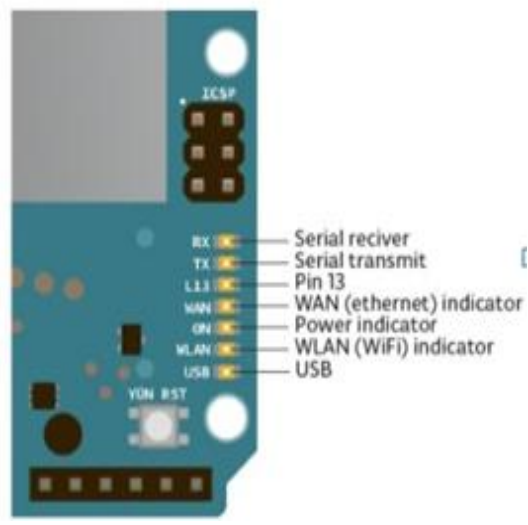

(b)

Figure 5. (a) Schematic of Arduino Yun, (b) Multiple statues indicator by LED [4].

The Yun is completely different from other Arduino boards' i.e. It is capable of communicating directly with the Linux operating system onboard and act as the minicomputer for the Arduino like in raspberry pi as shown in Fig. 4. In addition to Linux command, we can develop our own python scripts and modified scripts for strong communications. It depends on user point of view that which mode is utilized for data transfer i.e. by using Wi-Fi or by using Ethernet.

Now after this, all the data is acquired by the Arduino Yun (Fig. 5) which then transfers data to the cloud and controlled by data handling services like kaa, Watson etc. Admin performs several actions on the received data according to the user specification. If the level of water in the farm gets increased beyond certain critical level defined by the user, then Arduino Yun take its action to open the valve by activating relay which will release the water to the other farm. In summer every farmer faces inadequate amount of water which is not sufficient for growing of some crops. So this can be minimized by activating the dc motor which brings water from already filled water reservoir to the respective field which requires water. Based on the moisture level of the crop, water is providing in the form of droplets through pipes in every area. We can analyze all live information about our field by using Smartphone or tablet. We use kaa IoT platform for accessing and visualizing all the sensor data through cloud. Kaa is open-source IoT platform for flexible construction, organization, and integration of applications in the Internet of Things. Kaa will add lots of capabilities and features into connected products and smart devices. Kaa increases the speeds of the operation of devices and gathers data in sophisticated manner.

This platform is the combination of the several components of the server and the endpoint devices SDK which incorporated into any application according to the client.

Kaa provides easy delivery of event messages to each and every device which are connected. Data collection system: Kaa having its impermanent storage of various devices data records which is known as "logs". The kaa server can store logs into the file system. Kaa can utilize big data analysis and handling vendors like Hadoop, MongoDB for managing data in an effective manner. Profiling and grouping of data: Kaa consists of client-side and server-side which are the two part of endpoints profile. Client-side endpoint presents data to the Kaa resource manager and UI managing system to display data in an efficient manner. All the data received by Kaa server is controlled by Kaa server users via Administration dashboard User interface. Notifications delivery of message: Kaa has warning and alert system which used in delivering any message of predefined structure to the endpoints devices by the kaa server. Data distribution over the internet: Kaa perform the specific task on the obtained data which is sent by the client server and compare with standard data and give result and distribute over the internet.

Vinod Sukhadeve and Sahadev Roy "Advance Agro Farm Design With Smart Farming, Irrigation And Rain Water Harvesting Using Internet of Things," International Journal of Advanced Engineering and Management, Vol. 1, No. 1, pp.33-45, 2016. 
Transportation: At the end, to transfer the data from sensor world to display it on web UI. It has various supported transportation services like, MQTT, CoAP, Ethernet, Zigbee, XMPP, HTTP, Wi-Fi, and TCP, etc.

\section{a) Kaa supporting operation system, platform, and large data processing system}

Operating systems: Android, ios, Linux, ubuntu, windows, QNX. Hardware platforms: Intel Edison, Beagle bone, Raspberry Pi, Econais, Leaf labs, Texas Instruments, 8266 Supported data processing system. Hadoop, Mongo Db, Oracle, Cassandra Spark Couch base. The main sensors which are required for the design of an IoTbased system for agriculture are shown in Fig. 7. We require soil humidity sensor for periodically checking soil humidity content each day according to the natural calamities. The other is the temperature sensor, water level detector sensor for the checking of water content in the farm during winter, summer and rainy season. Now we will see how to overcome the effect of pest, animal on the crop production by using the ultrasound. We know that pests are the destructive insects or others animal that attacks crops, food, livestock, etc such as grasshoppers, locusts, lacewings, moths etc. All living things made up of water Molecule so it generates heat in all living things because of frequency produced by ultrasound As a result, more heat energy is produced in insects[5]. So RF treatment is used to treat over pest without any adverse effect on food quality and production and also a loss to the farmer.

Table 3: Response of Animals and other species to Ultrasound
\begin{tabular}{|c|c|c|}
\hline Sr No & Frequency Range & Application \\
\hline 1 & $10-143 \mathrm{kHz}$ & $\begin{array}{c}\text { Birds, pigeons, deer, cattle } \\
\text { and other wild animal }\end{array}$ \\
\hline 2 & $38-44 \mathrm{kHz}$ & $\begin{array}{c}\text { Arthropods like Spiders, } \\
\text { Mosquitoes, Cockroaches, } \\
\text { Spiders, Fleas, }\end{array}$ \\
\hline 3 & $52-60 \mathrm{kHz}$ & Reptiles like Lizards \\
\hline 4 & $60-72 \mathrm{kHz}$ & Rodent like Rats \\
\hline
\end{tabular}

So according to the application, we have to vary the knob of frequency selector which is available in ultrasound generator mentioned according to Table 3. These all sensor are transferring its data by using XBee module which is worked as the transmitter as well as the receiver. It is available in various types with their application such as XBee SX, XBee-PRO 900HP, XBee802.15.4, XBee DigiMesh 2.4, XBee-PRO 868 etc. The signal is received by the router then transmitted to the gateway. The gateway connects all the sensor devices to the internet. We can store data received by using the local server located near to the site. If we want to analyze the sensor data anywhere from the world, we need to store that data to the cloud, where we can handle and analyze the sensor data by using any internet compatible Smartphone or laptop or tablets. To communicate all the sensor network to the internet, different wireless technologies are used, depends on the application, the user needs to choose proper wireless communication technology.

\section{Comparison and analysis various wireless communication technology used in loT}

\section{a) Bluetooth}

Bluetooth is short range wireless technology that enables low-power wireless connections . Bluetooth helps in linking to the IoT devices, sensors, actuators via a mobile and tablet in most cases. The recently developed LowEnergy based Bluetooth which known as Bluetooth Smart is an important protocol used for IoT and data transfer applications. The range of Bluetooth smart is same as that of normal Bluetooth, but the only difference in their power utilization[7]. Smart Bluetooth is not really designed for data transfer. It is appropriate for small fragments of data. The BLE technology was originally introduced by mobile maker Nokia in 2006, but not able to be a part of original Bluetooth standard until 2010Standard.

\section{b) Zigbee}

It's like Bluetooth but having a larger range of operation .ZigBee having two versions i.e. PRO and ZigBee which are based on the IEEE 802.15 .4 protocol, which is working at $2.4 \mathrm{GHz}$ allowing applications that need frequent rare data interchange at reasonable data rates over a public IP within nearly 100m range[8]. The latest version i.e. ZigBee 3.0 is recently launched which is the combination of the numerous ZigBee standards incorporated into one single standard. ZigBee having Frequency of $2.4 \mathrm{GHz}$, they can transmit and receive data up to Range $10-100 \mathrm{~m}$ and transfer Data up to $250 \mathrm{kbps}$.

ZigBee 3.0 Certified Products

Vinod Sukhadeve and Sahadev Roy "Advance Agro Farm Design With Smart Farming, Irrigation And Rain Water Harvesting Using Internet of Things," International Journal of Advanced Engineering and Management, Vol. 1, No. 1, pp.33-45, 2016. 
AES1220 -ZigBee Smart Energy Metering DeviceTalkingPlug 11202- ZigBee Smart Energy Load Control DeviceSafePlug 1203- ZigBee Home Automation Mains Power Outlet

\section{c) Z-Wave}

Z-Wave is a technology which operates on low-power radio frequency basically intended for house automation including controlling lamp and various sensors [9]. This technology especially used for reliable communication between small information packets. It wires full mesh networks topology without any necessary of coordinator node hence can control up to 232 devices. Sigma Designs is the only single maker of Z-Wave chip rather than ZigBee and others which are having multiple makers. This communication protocol consist various devices which are identified by unique network ID. The Network ID is the general detection of all the different nodes belongs to one logical given Z-Wave network under consideration.

\section{d) 6LowPAN}

It is a wireless mesh network which uses low power technology in which each and every node has its separate 128 bits IPv6 address. Due to this, all the devices are identified on the network with their uniqueness. 6LowPAN support data encapsulation and header compression mechanism which are not found in protocols like ZigBee or Bluetooth[10]. It is specially built by the developer for home automation.

\section{e) Thread}

It is a new networking based IPv6 protocols basically use in the home automation environment. It is different from protocols like Bluetooth or ZigBee. Thread Protocol is launched by thread technology group, which gives free of cost services. It is mainly based on important protocol standards like IPv6, IEEE802.15.4, and 6LoWPAN which give realistic IP-based solution for the Internet of Things. The thread can handle up to 250 nodes which are connected in the mesh. The user needs to upgrade their devices firmware to run the thread on devices which are having previously IEEE802.15.4 standard.

\section{f) Wi-Fi}

It is the most often choice for many developers currently. Wi-Fi is generally called as wireless LAN. It is technology where high frequency RF wave required for transmission of data from one place to other. WiFi range is nearly $50 \mathrm{~m}$ between two places. This WI-FI technology works only on high frequency RF signals. For low frequency it fails to work correctly. Currently this protocol is used in office, college, IoT application and home network and also in many boards such as arduino yun, dragino wifi shield, raspberry pi. The most common standard used is $802.11 \mathrm{n}$, which generally found in homes and several IoT applications which consist of distant sensor connected to the microcontroller. Wi-Fi offers high data transfer rate in the range of hundreds of Mbps which is greater than Bluetooth. The operating frequency range of Wi-Fi is varied from $2.4 \mathrm{GHz}$ and $5 \mathrm{GHz}$.It can form duplex link efficiently over $50 \mathrm{~m}$. The most typical data rate offer by Wi-Fi is from 150-200Mbps, but 600 Mbps is maximum speed.

\section{g) Cellular}

The connection between IoT devices is possible with help of technology i.e. Unlicensed Mobile Access (UMA) and GAN-Generic Access Network which is diagrammatically shown in figure 8. UMA offer the services to the network through UMA technologies such as the wireless network. Firstly, UMA was designed to give a right to use to GSM/EDGE cellular technology through different UMA points. Nowadays this is developed under name of GAN (generic access networks). Unlicensed Mobile Access also provides flawless switching between cellular network and Wi-Fi protocol. This technology connects various technologies such as Wi-Fi, Bluetooth, and 802.11 to the cellular network. Therefore it is very helpful where we can connect our sensor directly to the cellular base station of the various spectrum companies. The use and application of mobile have grown rapidly around the world. With access to wireless connectivity from cellular network, there will be the possibility of effective and short range wireless connectivity.

\section{h) $\mathrm{NFC}$}

This communication technology provides effective and safest duplex communications among electronic sensor and devices, and specially designed for Smartphone and tablet, allowing customers to perform contactless fee transaction, to access digital content and connection between two electronic sensors. By using NFC services user Vinod Sukhadeve and Sahadev Roy "Advance Agro Farm Design With Smart Farming, Irrigation And Rain Water Harvesting Using Internet of Things," International Journal of Advanced Engineering and Management, Vol. 1, No. 1, pp.33-45, 2016. 
can transfer any confidential information from one place to another which are apart from distance less than nearly $4 \mathrm{~cm}$ away. The NFC has Frequency of $13.56 \mathrm{MHz}$ (ISM) and range of $10 \mathrm{~cm}$. The NFC can transfer data at the rate of $100-420 \mathrm{kbps}$.

\section{i) Sigfox}

The technology is Sigfox which is having the range in between Wi-Fi and cellular. Sigfox uses ISM bands, whose licenses is available at free of cost. Sigfox is used in many machines to machine applications that power on a small Ah capacity battery. Sigfox uses UNB known as Ultra Narrow Band technology. Sigfox can relatively handle data which needs data around 10 to 1,000 bps. It can consume only little microwatts power as compared to the cellular network.

\section{j) Neul}

The operating principle of Neul is same as that of Sigfox which is operating at $1 \mathrm{GHz}$ band and provides high-quality noiseless coverage. This protocol is cost efficient and consumes less power. The new communications technology is known as Weightless which is a new WAN networking technology created and gives full support for the IoT devices which are largely competing next to existing CDMA GPRS, LTE, and 3G solutions. Neul protocol has Data Rates up to some bps to 100-kilo bits per sec.

\section{k) Lora WAN}

This technology is analogous to some extent to Sigfox and Neul. This protocol is related to WAN applications and it is used to design WANs which consume low power. This helps in the design of cost-effective mobile duplex communication in this internet of things world. This technology is consuming low-power during operation and supporting huge networks with billions of devices. Lora WAN protocol transfers and receives data ranging from $0.3 \mathrm{kbps}$ to $50 \mathrm{kbps}$.

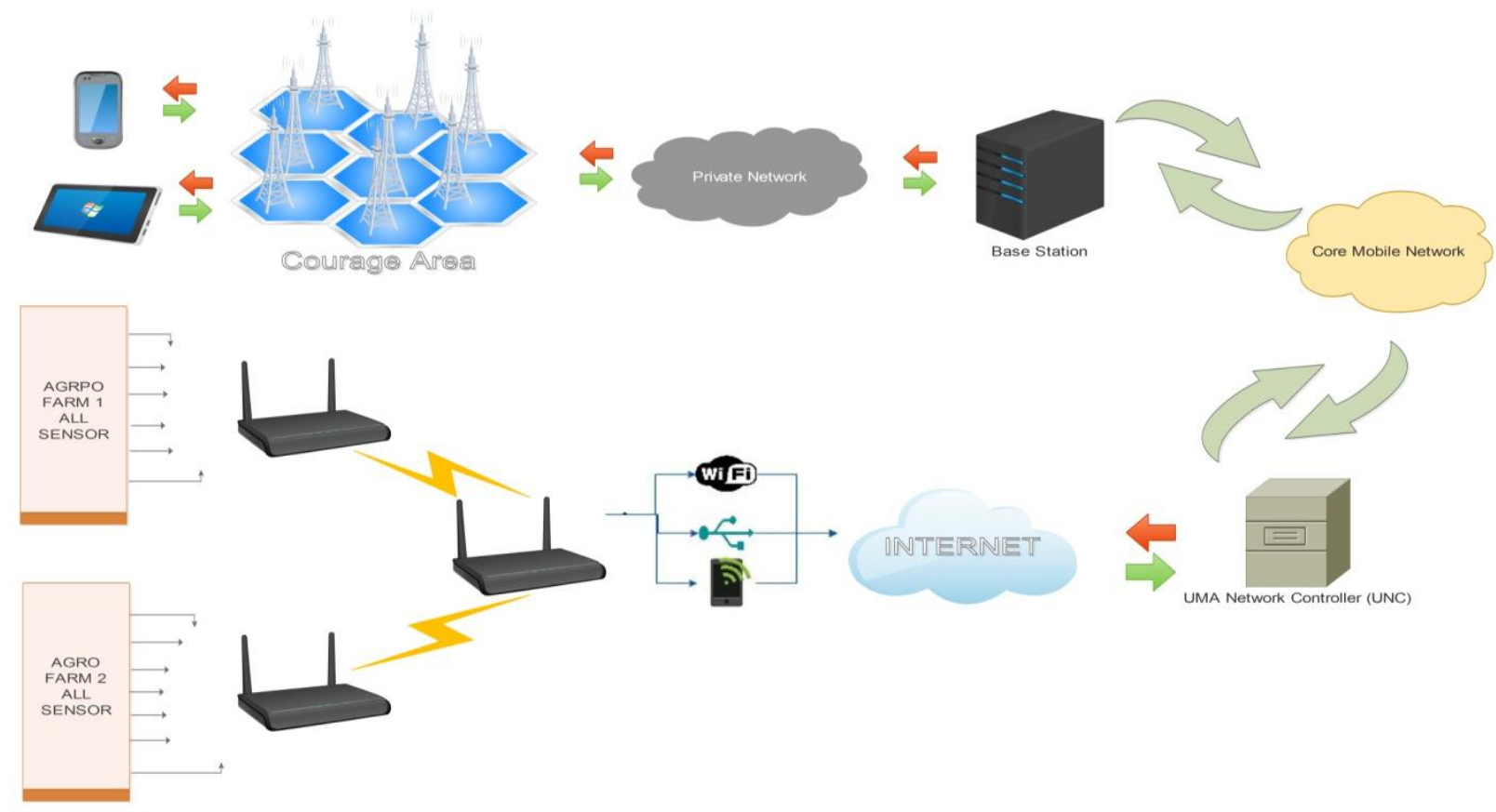

Figure 6. Connection of sensor devices with help of Unlicensed Mobile Access (UMA) and GAN-Generic Access Network.

\section{Design of network using netsim simulator and emulator}

Connection of sensor devices with help of Unlicensed Mobile Access (UMA) and GAN-Generic Access Network is shown in Fig. 6. It is a network tool which is used to simulate and emulate the user defined network design of for designing networks parameters. Netsim supports various technologies such as Wi-Max, WSN, TCP, Vinod Sukhadeve and Sahadev Roy "Advance Agro Farm Design With Smart Farming, Irrigation And Rain Water Harvesting Using Internet of Things," International Journal of Advanced Engineering and Management, Vol. 1, No. 1, pp.33-45, 2016. 
IP, and WLAN [11]. The Emulator which allows the users to connect user defined live application which is running on real devices. As a result, we can analyze the traffic in the real scenario. From this designed virtual $\mathrm{n} / \mathrm{w}$ numerous test is to be

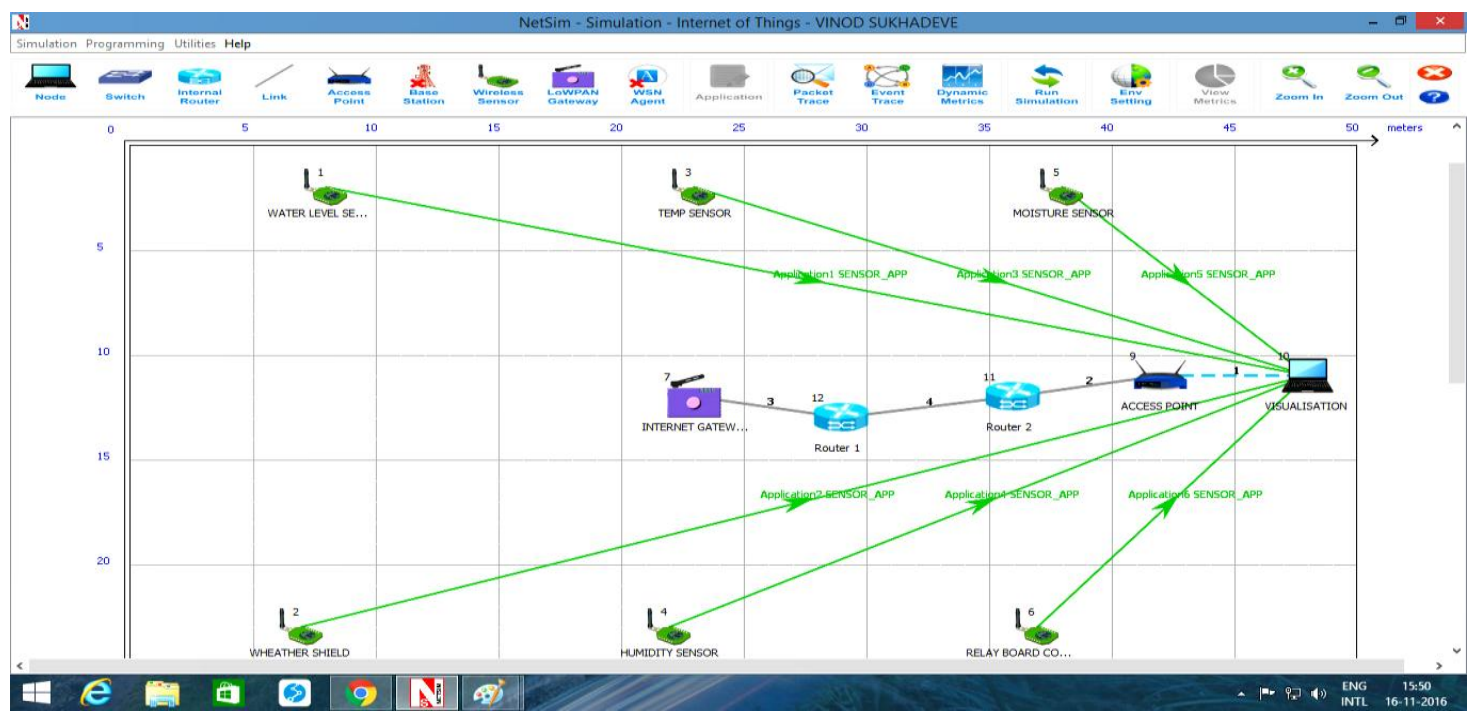

Figure 7. The snapshot of the design which shows the positioning of each sensor is as shown

performed repetitively such as packet loss bandwidth, link loss, overhead transmission etc. We assume the agriculture field size as $50 \mathrm{~m}$.The sensors are placed $15 \mathrm{~m}$ apart from each other, which sends their respective data to the cloud services through internet and display the information provided by a sensor. The snapshot of the design which shows the positioning of each sensor is as shown in Fig. 7 and its result analysis is shown in Fig. 8. A packet is a unit of data carried by a network, which consists of user data as well as the control information for the error correction and minimization. Typically, all the control information is embedded into headers and trailers. The packet which carries "payload" which is the information sends by the sensor. In WSN system, data which needs to be transferred from source to the destination is completely depends on the protocol we are using. So according to the application and user requirement, selection of protocol is depending. However, the "overhead" is the additional data required to send any packet which carries the payload. Due to overheads, unnecessary bandwidth gets wasted. TCP has more overhead with respect to UDP because of the special protocol features. For the above designed WSN network, we have performed simulation as well as emulation. The results are as shown in Table 4 .

Table 4. Link Metrics of IoT

\begin{tabular}{|c|c|c|c|c|c|c|}
\hline Link ID & $\begin{array}{c}\text { Packet } \\
\text { Transmitted }\end{array}$ & $\begin{array}{c}\text { Error } \\
\text { Packets }\end{array}$ & $\begin{array}{c}\text { Collided } \\
\text { Packets }\end{array}$ & Bytes Transmitted & Payload Transmitted & Overhead Transmitted \\
\hline 1 & 13735 & 11 & 0 & 873782 & 309800 & 563982 \\
\hline 2 & 6864 & 2 & 0 & 692544 & 309800 & 382744 \\
\hline 3 & 7406 & 0 & 0 & 568468 & 369932 & 198536 \\
\hline 4 & 7406 & 2 & 0 & 568460 & 369832 & 198628 \\
\hline 5 & 42580 & 0 & 11251 & 1367522 & 310200 & 1057322 \\
\hline
\end{tabular}

The link which connects the entire sensor via internet suffered by much simultaneous loss such as loss due to interference of noise and various types obstacles. For link quality estimation, we have to take many assumptions made in other environments. The losses due to obstacle sand interference, can lead to packet collisions and errors in the packets [12]. When two or more receiving stations try to attempt packet reception across any defined network, then packets collision occurs. When this type of collision occurs in network, the packets can be discarded or roll back to their originating places and then retransmitted after some defined time-lapse to avoid any further collision. The performance graph of the various links defined at the network emulated in NetSim tools is as shown bellows.

The each sensor is powered by the battery source, which is depends on two modes i.e. energy harvesting ON or energy harvesting OFF mode. For checking battery life of the sensor, we need to power the sensor without using energy harvesting mode. So, we can get the amount of sensor working life, and time requires to replacing the

Vinod Sukhadeve and Sahadev Roy "Advance Agro Farm Design With Smart Farming, Irrigation And Rain Water Harvesting Using Internet of Things," International Journal of Advanced Engineering and Management, Vol. 1, No. 1, pp.33-45, 2016. 
battery of the sensor. The bellows data obtained after emulating one of the sensors by giving some known input parameter.

Table 5: Power Model Metrics

\begin{tabular}{|c|c|c|c|c|c|}
\hline Devices ID & $\begin{array}{c}\text { Transmission } \\
\text { Energy Consumed }\end{array}$ & $\begin{array}{c}\text { Receiving Energy } \\
\text { Consumed }\end{array}$ & Idle Energy Consumed & Sleep Energy Consumed & $\begin{array}{c}\text { Total Energy } \\
\text { Consumed }\end{array}$ \\
\hline 1 & 07354 & 0.1433 & 29669.97 & 0.0000 & 29670.85 \\
\hline 4 & 0.0000 & 0.0000 & 0.0000 & 0.0000 & 0.0000 \\
\hline
\end{tabular}

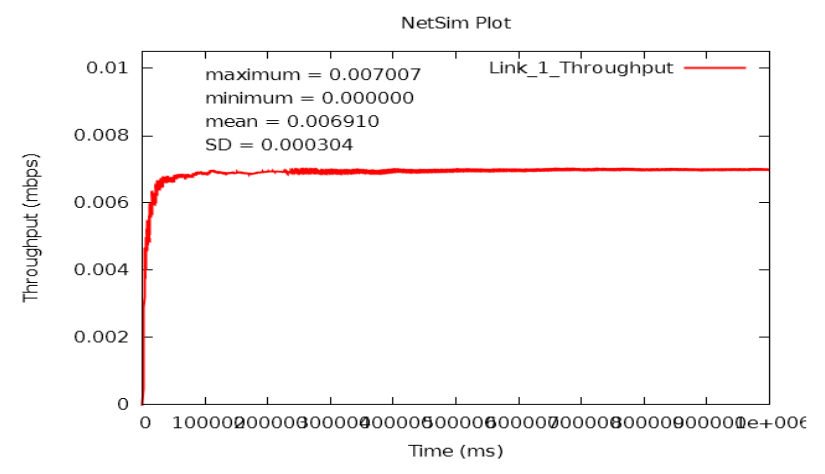

(a)

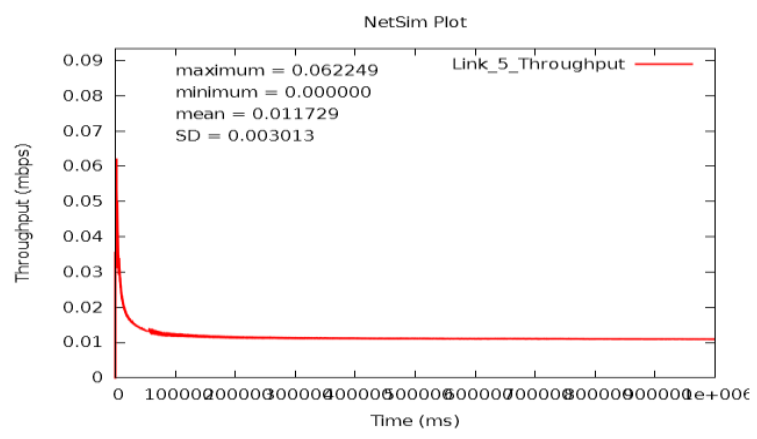

(c)

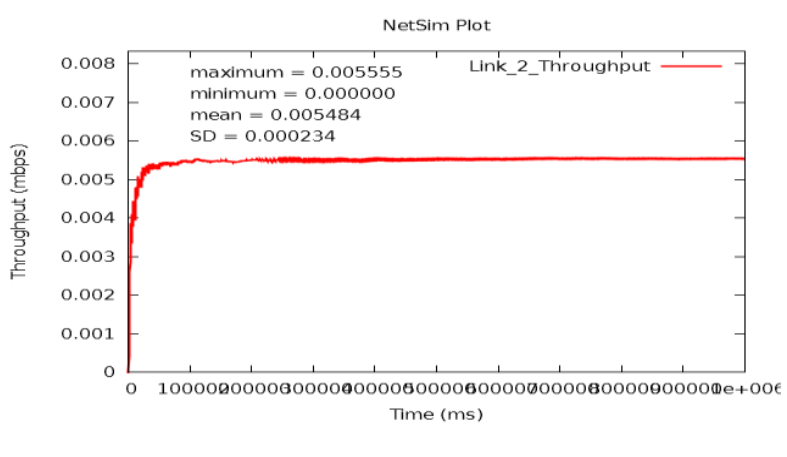

(b)

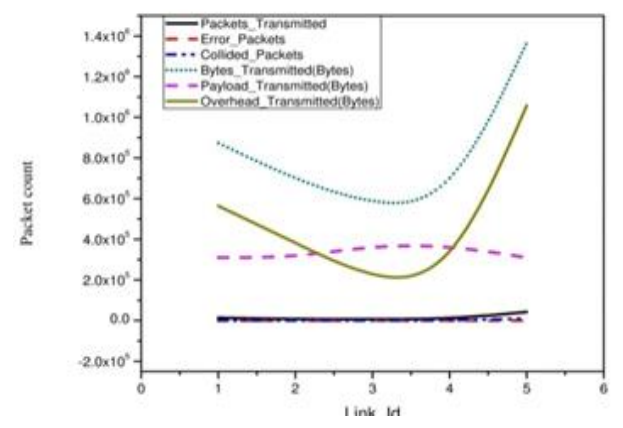

(d)

Figure 8. (a) Throughput of link 1, (b) Throughput of link 2, (c) Throughput of link 5, (d) Graph Between Various Links To Packets Counts By Considering Various Parameters Like Packet Transmitted, Error Packets, Collided Packets, Payload And Overhead Transmitted.

\section{Conclusion}

Based on the value obtained by emulating the designed network, following graphs are obtained by each link. This paper provides the solution to reduce the suicidal rate of farmers caused by the different problem faced by them. Using internet of things, the man makes agriculture more smart, effective and more productive. By using the mp3 shield, we can produce sounds of different animals used to threatened different animals which comes to destroy the crop. By using ultrasound of lower frequency, we can repel the pest which is destroying the crop so, farmers will not waste further money in buying pesticides and insecticide. Also, the health problems caused by the chemical used in pesticides through agriculture product will be minimizing to some extent. Also, we can predict early information about the status of battery used to power the sensor so that, we can replace the battery on time so that no sensor in the field gets off. Due to this no data packet loss feed by the sensor, therefore, an error will get reduced.

\section{References}

[1] D. Bandyopadhyay and S. Jaydip, "Internet of things: Applications and Challenges in Technology and Standardization," Wireless Personal Communications, vol. 58, no. 1, pp. 49-69, 2011.

Vinod Sukhadeve and Sahadev Roy "Advance Agro Farm Design With Smart Farming, Irrigation And Rain Water Harvesting Using Internet of Things," International Journal of Advanced Engineering and Management, Vol. 1, No. 1, pp.33-45, 2016. 
[2] R. v. d. Meulen. [Online]. Available: http://www.gartner.com/newsroom/id/3165317. [Accessed 1512 2016].

[3] D. ELLIOT, "http://www.cbsnews.com/," [Online]. Available: http://www.cbsnews.com/news/less-rainfallnot-the-only-risk-factor-in-widespread-drought-study-shows/. [Accessed 0310 2016].

[4] [Online]. Available: https://www.arduino.cc/en/Main/ArduinoBoardYun. [Accessed 14 december 2016].

[5] R. F. a. D. C. Sukun kim, "Reliable Transfer on Wireless Sensor Network," in First Annual IEEE Communications Society Conference, SECON 2004, 2004.

[6] R. v. d. Meulen, November 2015. [Online]. Available: http://www.gartner.com/newsroom/id/3165317.

[7] Friedman, K. A. Roy and Y. Krivolapov, "On Power and Throughput Tradeoffs of WiFii and Bluetooth in Smart Phones," IEEE Transactions on Mobile Computing, vol. 12, no. 7, pp. 1363-1376, 2013.

[8] P. Kinney, "Zigbee technology: Wireless control that simply works," in Communications Design Conference, 2003.

[9] Z. P. Liao, H. L. Wong, B. P. Yang and Y. F. Yuan, "A Transmitting Boundary for Transient Wave Analysis," Scientia Sinica, vol. 27, no. 10, pp. 1063-1076, 1984.

[10] I. K. Samaras, G. D. Hassapis and J. V. Gialelis, "A Modified DPWS Protocol Stack for 6LoWPAN-Based Wireless Sensor Networks," IEEE Transactions on Industrial Informatics, vol. 9, no. 1, pp. 209-221, 2013.

[11] "http://www.tetcos.com/," Tetcos. [Online]. [Accessed 11 2016].

[12] S. Kim, R. Fonseca and D. Culler, "Reliable Transfer on Wireless Sensor Networks," in Sensor and Ad Hoc Communications and Networks, 2004. IEEE SECON 2004. 2004 First Annual IEEE Communications Society Conference, 10.1109/SAHCN.2004.1381947.

Vinod Sukhadeve and Sahadev Roy "Advance Agro Farm Design With Smart Farming, Irrigation And Rain Water Harvesting Using Internet of Things," International Journal of Advanced Engineering and Management, Vol. 1, No. 1, pp.33-45, 2016. 